\title{
Nested Tail-Biting Convolutional Codes Construction for Short Packet Communications
}

\author{
Yaping $S U N^{1}$, Gaoqi DOU ${ }^{1}$, Mingliang $Y A N^{2}$ \\ ${ }^{1}$ School of Electronic Engineering, Naval University of Engineering, Jiefang Avenue 717, 430000 Wuhan,China \\ ${ }^{2}$ Unit 92118 of the Chinese People's Liberation Army, Qingleitou Road, 316000 Zhoushan,China
}

5103117015@email.ncu.edu.cn,hjgcqq@163.com,hjgcsyp@163.com

Submitted January 28, 2021 / Accepted May 11, 2021

\begin{abstract}
Tail-biting convolutional codes (TBCCs) have become a research hotspot in the short-block-length regime due to the growing interest in strong short packet communications with low latency and ultra-reliability. TBCCs can maintain the decoding performance without rate loss caused by the tailed bits in the traditional convolutional encoder, which also have good rate compatibility with better decoding performance than those of the iterative scheme for short block length codes. In this paper, a search algorithm is proposed to construct a set of rate-compatible TBCCs (RC-TBCCs) with consistent good frame error rate (FER) performance for fixed information length at various code rates. The algorithm considers the minimum distance profile of TBCCs. A set of RC-TBCCs is constructed for code rates from $1 / 3$ to $1 / 8$. The simulation results show that the proposed RC-TBCCs are superior to the LTE standard $R C$-TBCCs at different code rates.
\end{abstract}

\section{Keywords}

Short packet communications, tail-biting convolutional code (TBCC), nested rate-compatible TBCC (RC-TBCC), minimum distance profile

\section{Introduction}

In $5 \mathrm{G}$ system, channel coding technology is divided into three scenarios: EMBB, MMTC and URLLC [1]. Applications need to support requirements for different scenarios, involving machine communication, intelligent metering networks, remote command linking and messaging service [2], which require the channel coding scheme to support short packets from tens to hundreds of bits. One challenge is the design of short-block-length channel codes with good coding gain to support multiple data transmission rates.

TBCC is a short candidate code for URLLC standard in $5 \mathrm{G}$ communication [3], [4]. It eliminates the rate loss caused by tailed bits, meanwhile increasing the equivalent decoding depth through cyclic trellis network, which can avoid the performance degradation caused by decoding truncation for short convolutional codes. For short codes, TBCC can perform significantly better than binary and non-binary iterative coding schemes of Turbo and LDPC [5], [6]. The literatures studied the application of TBCC in short-length codes, and analyzed the performance of TBCC under different constraint lengths, block lengths and code rates in [7], [8]. L. Gaudio compared the performance of TBCCs with finite-length performance bounds and that of other channel codes [8]. Jingjing Liu proposed a construction method of rate-compatible short LDPC by puncturing [9].

For one-way communication or broadcast communication systems without retransmission, the channel codes should adapt according to source and channel conditions by sending additional incremental bits until the receiver can decode the message correctly, which can avoid the process of handshake and protocol used in the transformation of communication rates and realize the adaptive communication. RC-TBCCs have good rate compatible flexibility and mature coding/decoding algorithms [10], [11]. Rate-compatible codes are a group of code families in which the bits of high rate codes are included in the bits of low rate codes, and the encoding and decoding process can be realized by a single decoder. In the LTE standard, the repetition and puncturing method [12] is often used to realize the ratecompatible codes. However, the coding gain of the codes obtained by this method is limited when code rate is low, which cannot guarantee the efficient and reliable communication for various rates. Therefore, it is of great interest to design RC-TBCCs with consistent good performance for variable rates. This can be achieved by constructing the low-rate codes through appending incremental bits to highrate codes, namely, by nesting the code [13]. Reference [14] proposed an algorithm to construct nested RC-TBCCs which have consistently good FER performance for all information lengths and all compatible rates. However, it did not consider the RC-TBCCs with fixed information length. The multiple rate codes with fixed information length are often useful for coded cooperative communication. For example, high performance multi-rate codes are searched for the source-relay and the source-destination links with decode-forward relaying [15]. Moreover, there may be more than one set of TBCCs with optimal distance profile in one recursion, which can be used as mother codes 
in the next recursion. However, the reference didn't investigate this possibility further.

In this paper, a group of nested RC-TBCCs is designed, which have consistently good performance for fixed information length at various code rates. Based on the comprehensive consideration of the minimum distance profile and performance bounds of TBCCs, a search algorithm is proposed to construct nested RC-TBCCs. Besides, a group of nested RC-TBCCs is designed for code rates ranging from $1 / 3$ to $1 / 8$ with the same encoder and decoder structure. The simulation results show that the nested RCTBCCs proposed in this paper are superior to the LTE standard RC-TBCCs at different code rates.

\section{Minimum Distance Properties and Performance Bounds of TBCCs}

In reference [16], the definition of minimum distance of convolutional codes is given. Due to the tail-biting characteristic of TBCCs, the definition of the minimum distance of ordinary convolutional codes cannot be simply applied to TBCCs. In this section, the definition of the minimum distance and the performance bounds of TBCCs is given.

\subsection{The Minimum Distance of TBCCs}

The minimum distance of TBCCs is defined as the minimum Hamming distance between any two TBCCs. For linear block codes, the minimum distance equals the minimum weight of all codewords generated by any non-zero information sequence, e.g.

$$
\begin{aligned}
d_{\min } & =\min _{u^{\prime}, u^{\prime \prime}}\left\{d\left(v^{\prime}, v^{\prime \prime}\right): u^{\prime} \neq u^{\prime \prime}\right\} \\
& =\min _{u}\{w(v): u \neq 0\} \\
& =\min _{u}\{w(u G): u \neq 0\}
\end{aligned}
$$

where $v^{\prime}, v^{\prime \prime}$ and $v$ are the codes corresponding to information sequence $u$ ', $u$ ', and $u$ respectively.

Trellis paths of the codewords corresponding to nonzero information sequences fall into three categories, which are provided in Fig. 1 to Fig. 3. least twice.

Case (i): Trellis path that touches the all-zero path at

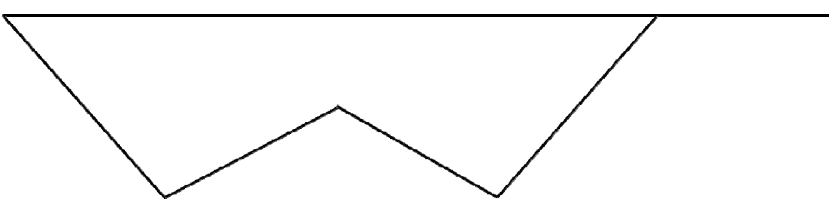

Fig. 1. Trellis path that touches the all-zero path at least twice.

Case (ii): Trellis path that touches the all-zero path only once.

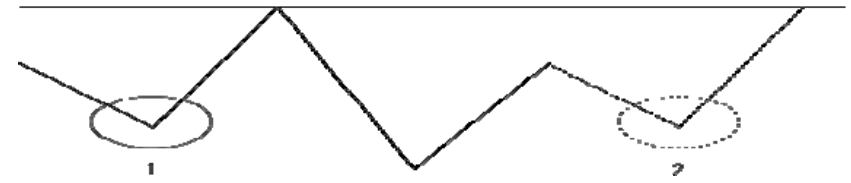

Fig. 2. Trellis path that touches the all-zero path only once.

Case (iii): Trellis path that has never touched the allzero path.

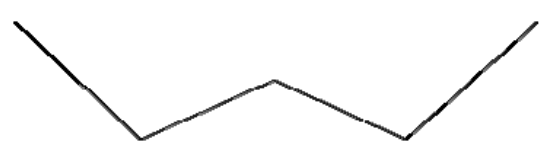

Fig. 3. Trellis path that has never touched the all-zero path.

The solid line in Fig. 2 is a trellis path that touches the all-zero path only once. Since the starting state of the TBCC is identical to its ending state, (1) in Fig. 2 can be shifted to position (2) to form a trellis path that touches the all-zero path twice.

From the above analysis, case (i) and case (ii) can be merged into one case, the codes of which are denoted as $C_{\mathrm{tz}}$. The minimum weight of $C_{\mathrm{tz}}$ generates from the path that splits and merges again later to the all-zero path. The free distance is also generated in this case. The codewords of case (iii) are denoted as $C_{\text {ntz }}$, and the minimum weight of $C_{\text {ntz }}$ generates from the path that has never touched the allzero path. When the code length is long enough, the minimum distance is generated in $C_{\mathrm{tz}}$. However, for short codes, the minimum distance may be generated in $C_{\text {ntz }}$, e.g.

$$
d_{\mathrm{min}, K}=\min \left\{\min _{u_{i} \neq 0}\left\{w\left(\mathrm{C}_{\mathrm{tz}, i}\right)\right\}, \min _{u_{j} \neq 0}\left\{w\left(\mathrm{C}_{\mathrm{ntz}, j}\right)\right\}\right\} .
$$

\subsection{The Performance Bounds of TBCCs}

For AWGN channel with binary input and continuous output, the FER performance bound of a linear code with BPSK modulation can be upper-bounded by [16]

$$
\begin{aligned}
P & \leq \sum_{d=d_{\min , K}}^{\infty} A_{d, K} Q\left(\sqrt{\frac{2 d E_{\mathrm{s}}}{N_{0}}}\right) \\
& \triangleq \sum_{d=d_{\min , K}}^{\infty} A_{d, K} \frac{1}{\sqrt{2 \pi}} \int_{\sqrt{\frac{2 d E_{\mathrm{s}}}{N_{0}}}}^{\infty} \exp \left(-\frac{y^{2}}{2}\right) \mathrm{d} y
\end{aligned}
$$

where $d$ is the weight of a codeword and $A_{d, K}$ is the number of weight- $d$ codewords when the information length is $K$, and $E_{\mathrm{s}} / N_{0}$ is the signal-to-noise ratio (SNR).

For high SNR, $Q\left(\sqrt{2 d E_{\mathrm{s}} / N_{0}}\right)$ decreases exponentially with the increase of $d$, so the performance bound is mainly determined by the first term of (3), e.g.

$$
\tilde{P} \approx A_{d_{\min , K}} Q\left(\sqrt{2 d_{\min , K} E_{\mathrm{s}} / N_{0}}\right)
$$


where $d_{\min , K}$ is the minimum weight of all the codewords, and $A_{d_{\min , K}}$ is the number of weight- $d_{\min , K}$ codewords with information length $K$. From (4), the performance bound of FER decreases exponentially with the increase of $d_{\min , K}$, and increases linearly with the increase of $A_{d_{\min , K}}$. This relationship indicates that the most important criterion to construct a good convolutional code is to maximize $d_{\min , K}$, and the secondary criterion is to minimize $A_{d_{\min , K}}$.

\section{Construction of Nested RC-TBCCs}

Rate-compatible capability is significantly important in many communication systems. To achieve rate compatibility, repetition and puncturing methods are commonly used in the LTE standard. However, this leads to limited FER performance for codes with low code rates. In the following, nested RC-TBCCs are introduced and a construction algorithm for nested RC-TBCCs is proposed to further improve FER performance at low code rates and achieve better FER performance at all compatible code rates.

\subsection{Nested RC-TBCCs}

Mother codes of rate $1 / n$ with generation polynomial $\mathbf{g}_{n}=\left(g_{1}, g_{2}, \ldots, g_{n}\right)$ can generate TBCCs of rate $1 /(n+1)$ by appending an additional generator $g_{n+1}$ to $\mathbf{g}_{n}$, e.g. $\mathbf{g}_{n+1}=\left(\mathbf{g}_{n}, g_{n+1}\right)$. Similarly, a set of nested RC-TBCCs $\mathbf{g}_{n+1}=\left(\mathbf{g}_{n}, \mathbf{g}_{n+1}, \ldots, \mathbf{g}_{n+l}\right)$ with code rates ranging from $1 / n$ to $1 /(n+l)$ can be obtained by recursively appending additional generators, namely nesting. In this set of codewords, all coded bits of the high rate codewords are included in the coded bits of the low rate codewords, so the nested RCTBCCs can be decoded by a single decoder.

\subsection{The Search Algorithm of Nested RC- TBCCs}

From Sec. 2, the most important criterion to construct a good TBCC is to maximize $d_{\min }$, and the secondary criterion is to minimize $A_{d_{\min }}$. Therefore, in order to achieve the optimal FER performance for fixed information length at all compatible code rates, the minimum distance profile $\left\{d_{\min }, A_{d_{\min }}\right\}$ must be optimized at all rate values. However, although the minimum distance profile of nested RCTBCCs at one code rate is optimal, the distance profile for other rates may not be optimal. Therefore, it is best to have some degree of tradeoff between the optimization of FER for all compatible code rates. Thus we introduce two concepts in the paper: $d_{\min }$ divergence with respect to the $d_{\text {min }}$ upper bound (MDD) and $A_{d_{\min }}$ divergence with respect to the $A_{d_{\min }}$ lower bound (AMDD), which will be analyzed next. The nested RC-TBCCs with minimized MDD and minimized AMDD would have consistently good performance for various compatible code rates. Therefore, the criteria of the search algorithm for nested RC-TBCCs can be denoted as
- optimized minimum distance profile $\left\{d_{\min }, A_{d_{\min }}\right\}$ and

- minimized MDD and

- minimized AMDD.

The explanations about MDD and AMDD introduced above are as follows.

For a given mother code $\mathbf{g}_{n_{\min }}=\left(g_{1}, g_{2}, \ldots, g_{n_{\min }}\right)$ with fixed information length, $N$ groups of nested RC-TBCCs are constructed through the nested method, in which a set of nested RC-TBCCs is denoted as $\left\{\mathbf{g}_{n_{\min }^{(j)}}, \mathbf{g}_{n_{\min }+1}^{(j)}, \cdots, \mathbf{g}_{n_{\max }}^{(j)}\right\}$ and thus the TBCCs with code rate $1 / n_{i}$ are denoted as $\left\{\mathbf{g}_{n_{i}}^{(1)}, \mathbf{g}_{n_{i}}^{(2)}, \cdots, \mathbf{g}_{n_{i}}^{(N)}\right\}$. We denote the minimum distance of $\mathbf{g}_{n_{i}}^{(j)}$ as $d_{\min , n_{i}}^{(j)}$ and the upper bound of $d_{\min }$ at code rate $1 / n_{i}$, e.g. $D_{\min , n_{i}}$, is given by

$$
D_{\min , n_{i}}=\max _{j=1}^{N}\left\{d_{\min , n_{i}}^{(j)}\right\} .
$$

$D_{\min , n_{i}}$ represents the largest $d_{\text {min }}$ of TBCCs at the given code rate $1 / n_{i}$ among these groups.

We define MDD ( $d_{\text {min }}$ divergence with respect to the upper bound of $\left.d_{\min }\right)$ as

$$
\Gamma^{(j)}=\sum_{n=n_{\min }}^{n_{\max }}\left(d_{\min , n}^{(j)}-D_{\min , n}\right)^{2} .
$$

$\Gamma^{(j)}$ measures the Euclidean distance between $d_{\min }$ of a set of nested RC-TBCCs and the $d_{\min }$ upper bound of all groups. The nested RC-TBCCs with smaller MDD have a higher probability to have $d_{\min }$ closer to the $d_{\min }$ upper bound, and thus it is more likely to have good FER performance when the code rate changes between $n_{\min }$ to $n_{\max }$.

We denote $A_{d_{\min }}$ corresponding to $\mathbf{g}_{n_{i}}^{(j)}$ as $A_{d_{\min }, n_{i}}^{(j)}$, in which $A_{d_{\min }}$ represents the number of weight- $d_{\min }$ paths in the trellis of TBCCs, and the lower bound of $A_{d_{\text {min }}}$, e.g. $A_{\text {min }, n_{i}}$, is given by

$$
A_{\min , n_{i}}=\min _{j=1}^{N}\left\{A_{d_{\min }, n_{i}}^{(j)}\right\} .
$$

$A_{\text {min, } n_{i}}$ represents the smallest $A_{d_{\min }}$ of TBCCs at the given code rate $1 / n_{i}$ among these groups.

Similarly, we define AMDD $\left(A_{d_{\min }}\right.$ divergence with respect to the lower bound of $A_{d_{\min }}$ ) as

$$
\mathrm{T}^{(j)}=\sum_{n=n_{\min }}^{n_{\max }}\left(A_{d_{\min }, n}^{(j)}-A_{\min , n}\right)^{2} .
$$

$\mathrm{T}^{(j)}$ measures the Euclidean distance between $A_{d_{\text {min }}}$ of a set of nested RC-TBCCs and the $A_{d_{\min }}$ lower bound of all groups. The nested RC-TBCCs with smaller AMDD have a higher probability to have $A_{d_{\min }}$ closer to the $A_{d_{\min }}$ lower bound, and thus it is more likely to have good FER performance when the code rate changes between $n_{\min }$ to $n_{\max }$.

Through the analysis above, nested RC-TBCCs with minimized MDD and minimized AMDD are more likely to achieve better FER performance at various code rates. 


\section{Algorithm 1}

Recursive Search of Nested RC-TBCCs with Consistent FER Performance for Various Compatible Code Rates

Input: mother code $\mathbf{g}_{n_{\min }}$ with constraint length of $m$, minimum code rate $1 / n_{\max }$, and information length $K$.

Output: groups of nested RC-TBCCs for code rate $1 / n_{\max } \leq r \leq 1 / n_{\min }$.

1: Initialize mother codes : $\Omega_{n_{\min }, 0} \leftarrow\left\{\mathbf{g}_{n_{\min }}\right\}$

$2:$ for $n=n_{\min }: n_{\max }-1$ do

3: for $i=1: I$ (the size of $\Omega_{n, 0}$ )

4: Generate a set of rate- $1 /(n+1)$ candidates $\Omega_{n+1,1}$ based on $\Omega_{n, 0}^{(i)}$.

5: Save the TBCCs with the largest $d_{\min }$ :

$$
\Omega_{n+1,2} \leftarrow \underset{g_{n+1} \in \Omega_{n+1,1}}{\arg \max }\left\{d_{\min }\right\} \text {. }
$$

6: Save the TBCCs with the smallest $A_{d_{\min }}$ :

$$
\Omega_{n+1,0} \leftarrow \underset{g_{n+1} \in \Omega_{n+1,2}}{\arg \min }\left\{A_{d_{\min }}\right\} \text {. }
$$

7: end for

8: end for

9: Save the TBCCs with minimized $d_{\min }$ divergence:

$$
\Omega_{3} \leftarrow \underset{g \in \Omega_{n_{\max }, 0}}{\arg \min }\{\Gamma\} .
$$

10: Save the TBCCs with minimized $A_{d_{\min }}$ divergence:

$$
\Omega_{\text {out }} \leftarrow \underset{g \in \Omega_{3}}{\arg \min }\{\mathrm{T}\} .
$$

Therefore, the algorithm description for constructing nested RC-TBCCs is provided in Algorithm 1. The construction begins with a mother code $\mathbf{g}_{n_{\min }}$ with constraint length $m$. In every recursion, a set of rate- $1 /(n+1)$ candidates is obtained by appending all the possible additional generator to one of the rate- $1 / n$ mother codes list $\Omega_{n, 0}$. E.g., for a list of mother codes $\Omega_{n, 0}=\left\{\mathbf{g}_{n}^{(1)}, \mathbf{g}_{n}^{(2)}, \cdots, \mathbf{g}_{n}^{(l)}\right\}$ with constraint length 6 , the generated candidates for $\Omega_{n, 0}^{(i)}=\mathbf{g}_{n}^{(i)}$ contain the nested RC-TBCCs as follows (in octal representation):

$$
\Omega_{n+1,1}=\left\{\left(\mathbf{g}_{n}^{(i)}, 100\right),\left(\mathbf{g}_{n}^{(i)}, 101\right), \cdots,\left(\mathbf{g}_{n}^{(i)}, 177\right)\right\} .
$$

To obtain the nested RC-TBCCs with optimal FER performance from the candidates of a mother code, we search for TBCCs with the largest $d_{\min }$ and smallest $A_{d_{\min }}$ (Step 5,6$)$. And so on, for each of the mother codes list. In the following recursion, we take $\Omega_{n+1,0}$ as the mother codes list, and repeat Step 3, 4, 5, 6. The recursion is performed until the minimum code rate $1 / n_{\max }$ is reached. Next, to obtain the nested RC-TBCCs with consistently good FER performance for all the compatible code rates, e.g. $1 / n_{\max } \leq r \leq 1 / n_{\min }$, we search for TBCCs with minimized MDD and minimized AMDD. Finally, all the nested RCTBCCs within $\Omega_{\text {out }}$ satisfy the criterions above, therefore we

\begin{tabular}{|c|c|c|c|c|c|c|}
\hline Code rate & \multicolumn{3}{|c|}{ Proposed } & \multicolumn{3}{c|}{ LTE-RC-TBCC } \\
\hline $1 / n$ & $g_{n}$ & $d_{\min }$ & $A_{d_{\min }}$ & $g_{n}$ & $d_{\min }$ & $A_{d_{\min }}$ \\
\hline 1 & 133 & - & - & 133 & - & - \\
\hline $1 / 2$ & 171 & - & - & 171 & - & - \\
\hline $1 / 3$ & 165 & 15 & 189 & 165 & 15 & 189 \\
\hline $1 / 4$ & 117 & 20 & 126 & 133 & 18 & 63 \\
\hline $1 / 5$ & 135 & 25 & 63 & 171 & 25 & 189 \\
\hline $1 / 6$ & 157 & 30 & 63 & 165 & 30 & 189 \\
\hline $1 / 7$ & 135 & 36 & 252 & 133 & 33 & 63 \\
\hline $1 / 8$ & 113 & 40 & 63 & 171 & 40 & 189 \\
\hline
\end{tabular}

Tab. 1. Nested RC-TBCCs and LTE-RC-TBCCs $(133,171,165)$.

can select any set of nested RC-TBCCs from $\Omega_{\text {out }}$ as the constructed nested RC-TBCCs.

\subsection{Optimized Generation Polynomial of Nested RC-TBCCs}

In this section, in order to compare the proposed nested RC-TBCCs with RC-TBCCs in LTE, we construct two groups of RC-TBCCs based on the mother code $\mathbf{g}_{3}=[133,171,165]$ according to Algorithm 1 and LTEmatching method, denoted as 'Proposed' and 'LTE-RCTBCC'. The solution is still valid for other mother codes. But due to the limited space, we discuss only one of them. As shown in Tab. $1, d_{\min }$ and $A_{d_{\min }}$ are obtained according to the minimum distance definition of TBCCs in Sec. 2 and compared with LTE-RC-TBCCs (information length 64 bit). It can be seen from Tab. 1 that $d_{\min }$ of nested RCTBCCs is greater than that of LTE-RC-TBCCs at code rates $1 / 4$ and $1 / 7$, and equal to that of LTE-RC-TBCCs at other code rates. However, at these other code rates, $A_{d_{\min }}$ is less than that of LTE-RC-TBCCs. According to (3) and (4), the performance bound of FER decreases exponentially with the increase of $d_{\min }$, and increases linearly with the increase of $A_{d_{\min }}$. Consequently, the influence of $d_{\min }$ on FER performance is dominant. Therefore, it can be seen from Tab. 1 that the proposed nested RC-TBCCs would perform better than LTE-RC-TBCCs regarding consistent FER performance for all compatible code rates.

\section{Decoder of Nested RC-TBCCs}

It can be seen from the proposed construction method in Sec. 3 that all the coded bits in high rate codewords of nested RC-TBCCs are included in the coded bits of low rate codewords, that is, the high rate codewords can be obtained by deleting the last few bits of the low rate codewords. Therefore, a set of nested RC-TBCCs has the same trellis diagram and the receiver can use a single decoder to decode nested RC-TBCCs for all the compatible rates. In this section, a single decoder is proposed to decode nested RC-TBCCs. The principle of this method is expressed in Fig. 4.

The decoder of nested RC-TBCCs can be achieved by appending zeros to each frame of the received TBCCs to 


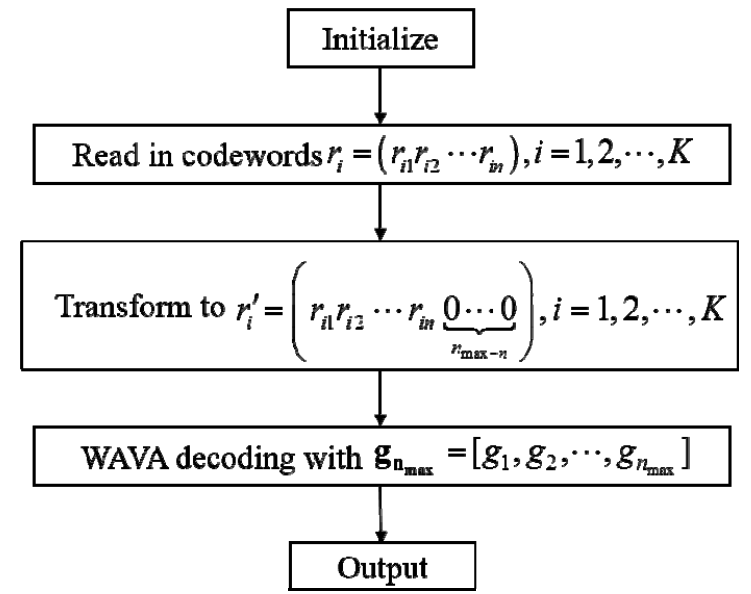

Fig. 4. Decoding flow chart of nested RC-TBCCs.

achieve maximum code rate $1 / n_{\max }$ which can be decoded by $\mathbf{g}_{n_{\max }}$. That's because a set of nested RC-TBCCs shares the same trellis diagram and the appended zeros can eliminate the influence of the last $n_{\max }-n$ generators on the path metric when using WAVA decoding. Therefore, nested RC-TBCCs decoding can be implemented through a traditional WAVA decoder with only a slight conversion of the received information.

\section{The Simulation Results}

In this section, the FER performance of nested RCTBCCs is evaluated through MATLAB simulation, in which the modulation mode is BPSK, the channel is AWGN channel, and the information length is 64 bits. At the receiving, a WAVA decoder with a maximum number of iterations of 5 is used for decoding. When calculating FER, at least 100 error frames are collected under different SNR.

FER performance of nested RC-TBCCs and LTE-RCTBCCs with code rates of $1 / 4,1 / 6$ and $1 / 7$ were simulated over a range of $E_{\mathrm{b}} / N_{0}$ ranging from $0.5 \mathrm{~dB}$ to $5 \mathrm{~dB}$, as shown in Fig. 5, Fig. 6, and Fig. 7. In the simulation 64-bit information is randomly generated. As we can see from these figures, the proposed nested RC-TBCCs have about $0.22 \mathrm{~dB}$ coding gain over LTE-RC-TBCCs at FER $=10^{-5}$ for code rate $1 / 4$. For code rates $1 / 6$ and $1 / 7$, the proposed nested RC-TBCCs have about $0.15 \mathrm{~dB}-0.2 \mathrm{~dB}$ coding gain over LTE-RC-TBCCs at FER $=10^{-5}$. What's more, Figure 8 shows the comparison of FER performance between the proposed nested RC-TBCCs and LTE-RC-TBCCs over a range of code rates ranging from $1 / 3$ to $1 / 8$ for $E_{\mathrm{b}} / N_{0}=$ $3 \mathrm{~dB}$ and $E_{\mathrm{b}} / N_{0}=4 \mathrm{~dB}$ (denoted as 'Proposed ( $\left.3 \mathrm{~dB}\right)$ ' and 'Proposed $(4 \mathrm{~dB})$ '). It can be seen from the figure that FER of the proposed nested RC-TBCCs is lower than that of LTE-RC-TBCCs for all compatible code rates, and the FER performance of the proposed nested RC-TBCCs is getting better and better with the increase of code rate.

Therefore, the simulation results show the superiority of the proposed nested RC-TBCCs over the LTE-RCTBCCs, which verifies the effectiveness of the proposed algorithm in the paper. For fading channel, we can obtain similar performance for the proposed nested RC-TBCCs. Due to the limited space, the simulation results aren't listed in the paper.

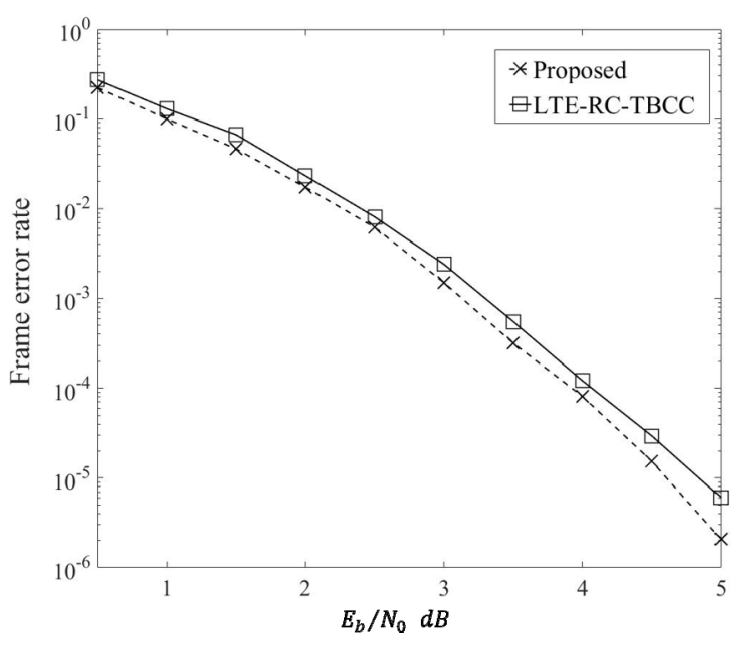

Fig. 5. FER of nested RC-TBCCs and LTE-RC-TBCCs at code rate $1 / 4$.

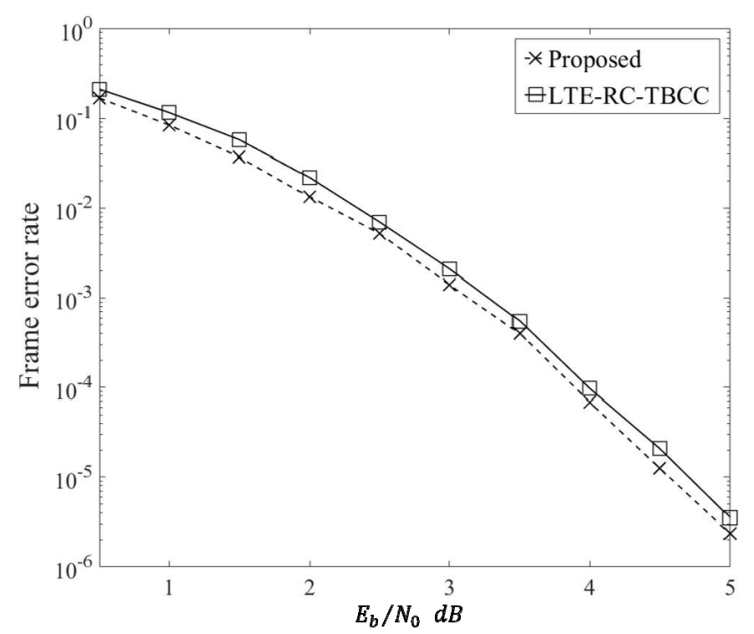

Fig. 6. FER of nested RC-TBCCs and LTE-RC-TBCCs at code rate $1 / 6$.

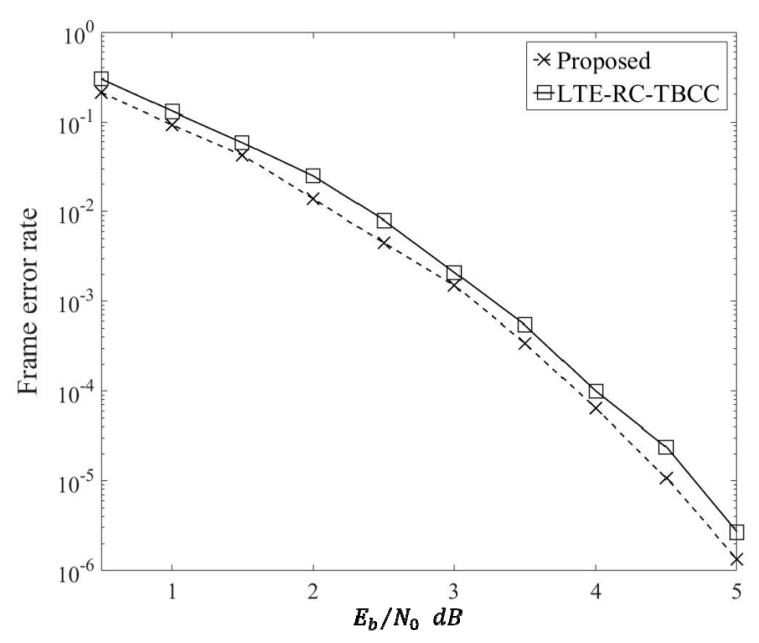

Fig. 7. FER of nested RC-TBCCS and LTE-RC-TBCCS at code rate $1 / 7$. 


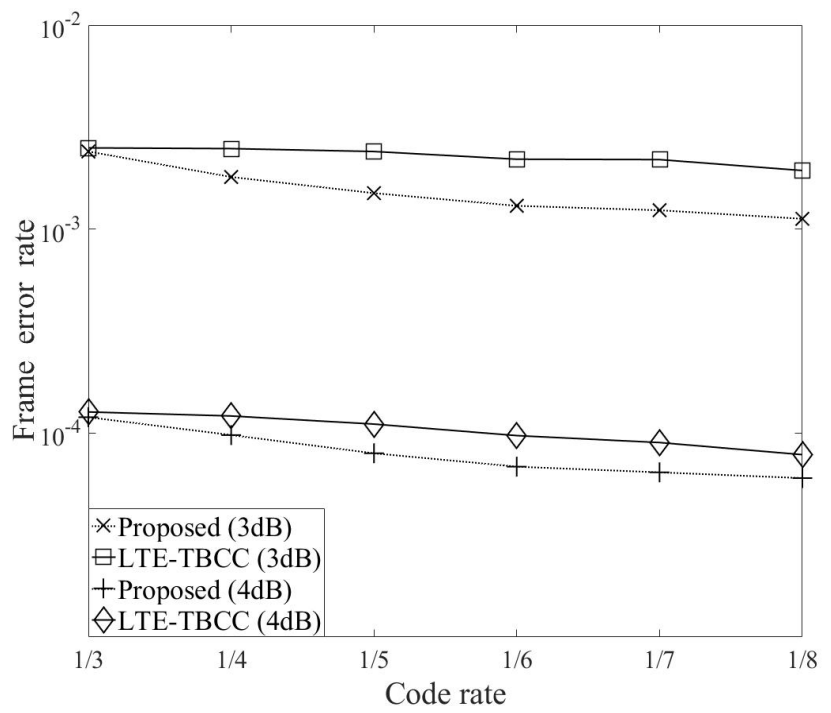

Fig. 8. FER of nested RC-TBCCs and LTE-RC-TBCCs at various code rates.

\section{Conclusion}

In this paper, a set of nested RC-TBCCs with consistent good FER performance is constructed, and a single decoder for the proposed nested RC-TBCCs is proposed. A set of nested RC-TBCCs ranging from $1 / 3$ to $1 / 8$ based on the proposed searching algorithm is given, and the FER performance of the proposed nested RC-TBCCs and LTERC-TBCCs are compared through MATLAB simulation. The simulation results show that the proposed nested RCTBCCs are superior to LTE-RC-TBCCs at various compatible rates and the FER performance of the proposed nested RC-TBCCs is getting better and better with the increase of code rate.

\section{Acknowledgments}

Sponsored by the National Natural Science Foundation of China (authorization number 61871473).

\section{References}

[1] BAE, J. H., ABOTABL, A., LIN, H. P., et al. An overview of channel coding for $5 \mathrm{G}$ NR cellular communications. APSIPA Transactions on Signal and Information Processing, 2019, vol. 8, p. 1-14. DOI: 10.1017/ATSIP.2019.10

[2] AHAMED, M., FARUQUE, S. $5 G$ Backhaul: Requirements, Challenges, and Emerging Technologies. Chapter 4 in Haidine, A., Aqqal, A. (eds.) Broadband Communications Networks - Recent Advances and Lessons from Practice, 2018. DOI: 10.5772/intechopen.78615

[3] SYBIS, M., WESOLOWSKI, K., JAYASINGHE, K., et al. Channel coding for ultra-reliable low-latency communication in 5G systems. In 2016 IEEE 84th Vehicular Technology Conference (VTC-Fall). Montreal (QC, Canada), 2016, p. 1-5. DOI: 10.1109/VTCFall.2016.7880930
[4] LIGURGO, M. IoT for 5G: Candidate Coding Schemes. Thesis, Politecnico di Torino, 2018, p. 1-66.

[5] LIAN BO Performance and Decoding Complexity Analysis of Short Binary Codes. Thesis, University of Toronto, 2019, p. 15-29.

[6] SHIRVANIMOGHADDAM, M., MOHAMMADI, M. S., ABBAS, R., et al. Short block-length codes for ultra-reliable low latency communications. IEEE Communications Magazine, 2018, vol. 57, no. 2, p. 130-137. DOI: 10.1109/MCOM.2018.1800181

[7] COSKUN, M. C., DURISI, G., JERKOVITS, T., et al. Efficient error-correcting codes in the short blocklength regime. Physical Communication, 2019, vol. 34, p. 66-79. DOI: 10.1016/j.phycom.2019.03.004

[8] GAUDIO, L., NINACS, T., JERKOVITS, T., et al. On the performance of short tail-biting convolutional codes for ultrareliable communications. In Proceedings of the 11th International ITG Conference on Systems, Communication and Coding (SCC). Hamburg (Germany), 2017, p. 1-6.

[9] JINGJING LIU, DE LAMARE, R. C. Rate-compatible LDPC codes with short block lengths based on puncturing and extension techniques. AEU-International Journal of Electronics and Communications, 2015, vol. 69, no. 11, p. 1582-1589. DOI: $10.1016 /$ j.aeue.2015.07.008

[10] KIM, J., TAK, J., KWAK, H., et al. A new list decoding algorithm for short-length TBCCs with CRC. IEEE Access, 2018, vol. 6, p. 35105-35111. DOI: 10.1109/ACCESS.2018.2847348

[11] RAVIV, T., SCHWARTZ, A., BEERY, Y. Deep Ensemble of Weighted Viterbi Decoders for Tail-Biting Convolutional Codes. P. 1-8. [Online] Available at: https://arxiv.org/abs/2009.02591

[12] ETSI TS 136.212 V15.3.0 LTE; Evolved Universal Terrestrial Radio Access (E-UTRA) Multiplexing and Channel Coding (3GPP TS 36.212 version 15.3.0 release 15), Jan. 2018.

[13] JORDAN, R., JOHANNESSON, R., BOSSERT, M. On nested convolutional codes and their application to woven codes. IEEE Transactions on Information Theory, 2004, vol. 50, no. 2, p. 380-384. DOI: 10.1109/TIT.2003.822612

[14] WU, X., YANG, L., YUAN, J., et al. Rate-compatible tail-biting convolutional codes for M2M communications. IEEE Communications Letters, 2017, vol. 22, no. 3, p. 482-485. DOI: 10.1109/lcomm.2017.2787054

[15] LIU, L., ZHOU, W., ZHOU, S. Nonbinary multiple rate QCLDPC codes with fixed information or block bit length. Journal of Communications and Networks, 2012, vol. 14, no. 2, p. 429-433. DOI: $10.1109 / \mathrm{JCN} .2012 .6292249$

[16] LIN, S., COSTELLO, D. J. Error Control Coding: Fundamentals and Applications. 2nd ed. Englewood Cliffs (NJ, USA): PrenticeHall, 2004. ISBN: 978-0130426727

\section{About the Authors ...}

Yaping SUN was born in Suzhou, China in 1995. She received a Bachelor of Engineering degree from the Naval University of Engineering in 2016, where she is currently pursuing a Master's degree. Her research interests include wireless communications, channel coding, and short block communications.

Gaoqi DOU (corresponding author) was born in Shanxi Province, China in 1981. He received the Ph.D. degrees from the Naval University of Engineering in 2009. His research interests include channel coding technology in wireless communication. 
Mingliang YAN was born in Jilin Province, China in 1993.

He received a Bachelor of Engineering degree from the
Naval University of Engineering in 2016. His research interests include channel coding and so on. 